\title{
On the Nonlinear Impulsive $\Psi$-Hilfer Fractional Differential Equations
}

\author{
Kishor D. Kucche ${ }^{a}$, Jyoti P. Kharade ${ }^{a}$ and \\ J. Vanterler da C. Sousa ${ }^{b}$ \\ ${ }^{a}$ Department of Mathematics, Shivaji University, \\ Kolhapur-416 004, Maharashtra, India \\ ${ }^{b}$ Department of Applied Mathematics, Imecc-Unicamp, \\ 13083-859, Campinas, SP, Brazil \\ E-mail(corresp.): kdkucche@gmail.com \\ E-mail: jyoti.thorwe@gmail.com \\ E-mail: ra160908@ime.unicamp.br
}

Received October 31, 2019; revised August 28, 2020; accepted August 28, 2020

\begin{abstract}
In this paper, we consider the nonlinear $\Psi$-Hilfer impulsive fractional differential equation. Our main objective is to derive the formula for the solution and examine the existence and uniqueness of solutions. The acquired results are extended to the nonlocal $\Psi$-Hilfer impulsive fractional differential equation. We gave an applications to the outcomes we obtained. Further, examples are provided in support of the results we got.
\end{abstract}

Keywords: $\Psi$-Hilfer fractional derivative, fractional differential equations, impulsive, nonlocal, existence and uniqueness, fixed point theorem.

AMS Subject Classification: 26A33; 34A08; 34A12; 34G20.

\section{Introduction}

The fractional differential equations (FDEs) over the years have been the object of investigation by many researchers $[1,4,14,19,22,24,37,39]$. Eminent mathematicians working in the field of FDEs has been exhibiting critical and fascinating outcomes throughout the years that contribute significantly to the mathematical analysis of FDEs, few of them are O'Regan [2], Agarwal [3], Balachandran, Trujillo [8], Benchohra [9], Diethelm [15], Feckan [17], Guo [18], Kilbas [19], Mophou [23], JinRong Wang [34], Wang [36] and Zhou [39].

Copyright (c) 2020 The Author(s). Published by VGTU Press

This is an Open Access article distributed under the terms of the Creative Commons Attribution License (http://creativecommons.org/licenses/by/4.0/), which permits unrestricted use, distribution, and reproduction in any medium, provided the original author and source are credited. 
The fact is that certain natural phenomena by means of fractional differential equations are modeled and allows to better describe the real situation of the problem compared to the problem modeled by means of differential equations of whole order. In this direction, the subject has picked up strength and interest of the researchers, since the fractional derivatives allows the variation of the order of the differential equation that is straightforwardly associated with the solution of FDEs. Recently, Sousa et al. [31] presented a fractional mathematical model by means of the time-fractional diffusion equation, which describes the concentration of nutrients in the blood and allows analyzing the solution of the model, better than the integer case. Other mathematical modeling and its analysis in the form differential equations with fractional order derivative can be found in $[13,16,21]$.

The existence, uniqueness, stability along with other qualitative properties of solutions for various kinds of FDEs without and with impulsive conditions (instantaneous and non-instantaneous) have been investigated in [9, 28, 29, 30, $32,36]$. The FDEs with impulsive effect play vital role in modeling real world physical phenomena involving in the study of population dynamics, biotechnology and chemical technology. Advancement in the theory of impulsive differential equations and its applications in the real world phenomena have been marvelously given in the monographs of Bainov and Simeonov [7], Benchohra et al [10] and Samoilenko and Perestyuk [25].

In 2009, Benchohra and Slimani [12] investigated various criterion for the existence of solutions for a class of initial value problems for impulsive fractional differential equations given by

$$
\left\{\begin{array}{l}
{ }^{c} D^{\mu} y(t)=f(t, y(t)), t \in[0, T], t \neq t_{k}, \\
\left.\Delta y\right|_{t=t_{k}}=I_{k}\left(y\left(t_{k}^{-}\right)\right) \\
y(0)=y_{0} \in \mathbb{R}
\end{array}\right.
$$

where ${ }^{c} D^{\mu}(\cdot)$ is the Caputo fractional derivative of order $0<\mu \leq 1, f$ : $[0, T] \times \mathbb{R} \rightarrow \mathbb{R}$ is a given function, $I_{k}: \mathbb{R} \rightarrow \mathbb{R}, k=1, \ldots, m, 0=t_{0}<t_{1}<$ $\cdots<t_{m}<t_{m+1}=T,\left.\Delta y\right|_{t=t k}=y\left(t_{k}^{+}\right)-y\left(t_{k}^{-}\right), y\left(t_{k}^{+}\right)=\lim _{h \rightarrow 0^{+}} y\left(t_{k}+h\right)$ and $y\left(t_{k}^{-}\right)=\lim _{h \rightarrow 0^{-}} y\left(t_{k}+h\right), k=1,2, \ldots, m$.

Benchohra and Seba [11] extended the study of existence for impulsive FDEs (1.1) in the Banach spaces. The following year, Benchohra and Berhoun [9], investigated sufficient conditions for the existence of solutions for impulsive FDEs with variable times.

In [17], Feckan et al. with the help of the examples it is demonstrated that the formula for the solutions of fractional impulsive FDEs (1.1) considered in the few referred papers in [17] were incorrect. They have derived the valid formula for the solution of impulsive FDEs (1.1) involving Caputo derivative and investigated the existence results for (1.1) using Banach contraction principle and Leray-Schauder theorem. In another interesting paper [37], Wang and coauthor presented the idea of piecewise continuous solutions for Caputo fractional impulsive Cauchy problems and impulsive fractioanl boundary value problem. They acquired existence and uniqueness of solution and furthermore determined data dependence and Ulam stabilities of solutions by means 
of generalized singular Gronwall inequalities. For recent studies on existence, uniqueness and Ulam-Hyers stabilty of solutions for various class of impulsive FDEs , we refer the work of Ali et al. [5], Shah et al. [26] and Wang et al. [34].

In any case, it is advantageous to utilize more broad fractional derivatives in which they hold a wide class of fractional derivatives as particular cases including the traditional ones of Caputo and Riemann-Liouville (RL). Sausa et al. [27] introduced the $\Psi$-Hilfer derivative operator ${ }^{H} \mathbf{D}_{a^{+}}^{\mu, \nu ;}$ that includes number of well known derivatives including widely used Riemann-Liouville and Caputo derivative. The advantage of studying the nonlinear FDEs involving $\Psi$ Hilfer derivative is that it preserves the investigated property of a FDEs with other fractional derivative operators listed in [27] which are particular cases of $\Psi$-Hilfer derivative. The fundamental study pertaining to existence and uniqueness and stability properties of the solution of FDEs involving $\Psi$-Hilfer derivative can be found in $[14,20,29,30,32,33]$.

Motivated by the works highlighted above, in the present paper, we consider the following impulsive $\Psi$-Hilfer fractional differential equation (impulsive $\Psi$ HFDE ) with initial condition

$$
\begin{aligned}
& { }^{H} \mathbf{D}_{a^{+}}^{\mu, \nu ; \Psi} u(t)=f(t, u(t)), t \in \mathcal{J} \backslash\left\{t_{1}, t_{2}, \ldots, t_{m}\right\}, \mathcal{J}=[a, T], T>a, \\
& \Delta \mathbf{I}_{a^{+}}^{1-\varrho ; \Psi} u\left(t_{k}\right)=\zeta_{k} \in \mathbb{R}, k=1,2, \ldots, m, \\
& \mathbf{I}_{a^{+}}^{1-\varrho ; \Psi} u(a)=\delta \in \mathbb{R}
\end{aligned}
$$

where $0<\mu<1,0 \leq \nu \leq 1, \varrho=\mu+\nu-\mu \nu,{ }^{H} \mathbf{D}_{a^{+}}^{\mu, \nu ;}(\cdot)$ is the $\Psi$ Hilfer fractional derivative of order $\mu$ and type $\nu, \mathbf{I}_{a^{+}}^{1-\varrho ; \Psi}$ is left sided $\Psi$-RL fractional integral operator, $a=t_{0}<t_{1}<t_{2}<\cdots<t_{m}<t_{m+1}=T$, $\Delta \mathbf{I}_{a^{+}}^{1-\varrho ; \Psi} u\left(t_{k}\right)=\mathbf{I}_{a^{+}}^{1-\varrho ; \Psi} u\left(t_{k}^{+}\right)-\mathbf{I}_{a^{+}}^{1-\varrho ; \Psi} u\left(t_{k}^{-}\right), \mathbf{I}_{a^{+}}^{1-\varrho ; \Psi} u\left(t_{k}^{+}\right)=\lim _{\epsilon \rightarrow 0^{+}} \mathbf{I}_{a^{+}}^{1-\varrho ; \Psi} u\left(t_{k}+\epsilon\right)$ and $\mathbf{I}_{a^{+}}^{1-\varrho ; \Psi} u\left(t_{k}^{-}\right)=\lim _{\epsilon \rightarrow 0^{-}} \mathbf{I}_{a^{+}}^{1-\varrho ; \Psi} u\left(t_{k}+\epsilon\right)$.

The main purpose of the paper is to derive the formula for the solution of Equations(1.2)-(1.4). We investigate the results pertaining to the existence and uniqueness of solutions for the impulsive $\Psi$-HFDEs Equations (1.2)-(1.4) through the equivalent fractional integral that we obtained. Further, we extend the obtained results to the non-local impulsive $\Psi$-HFDEs.

We highlight here a rigorous analysis of Equations(1.2)-(1.4) regarding the main results and advantages obtained in this paper:

- With $\Psi(t)=t$ and taking the limits $\nu \rightarrow 0$ and $\nu \rightarrow 1$ of the Equations(1.2)-(1.4), we obtain the respective special cases for the differential equations, that is, the classical fractional derivatives of Riemann-Liouville and Caputo, respectively. In addition to the integer case, by choosing $\mu=$ 1. These are two special cases of fractional derivatives. However, a wide class of fractional derivatives can be obtained from the choice of the parameters $\nu$ and $\Psi(t)$;

- Since it is possible to obtain a wide class of derivatives from the choice of $\nu$ and $\Psi(t)$; consequently, it is also possible to obtain a class of fractional differential equations with their respective fractional derivatives, as particular cases; 
Organization of Paper: In Section 2, some definitions and results that are important for the development of the paper have been provided via Lemmas and Theorems. In Section 3, we present a representation formula for the solution, i.e., we show that the problem (1.2)-(1.4) is equivalent to the Volterra fractional integral equation. In Section 4, we investigated the existence and uniqueness of the solution of impulsive $\Psi$-HFDE. In Section 5 , we will investigate the existence and uniqueness of a nonlocal impulse $\Psi$-HFDE. Finally, we close the paper with proposed future work.

\section{Preliminaries}

In this section, we introduce preliminary facts that are utilized all through this paper. Let $\Psi \in C^{1}(\mathcal{J}, \mathbb{R})$ an increasing function such that $\Psi^{\prime}(x) \neq 0, \forall x \in \mathcal{J}$.

Definition 1. [19] The $\Psi$-Riemann fractional integral of order $\mu>0$ of the function $h$ is given by

$$
\mathbf{I}_{a+}^{\mu ; \Psi} h(t):=\frac{1}{\Gamma(\mu)} \int_{a}^{t} \mathcal{L}_{\Psi}^{\mu}(t, \sigma) h(\sigma) d \sigma, \mathcal{L}_{\Psi}^{\mu}(t, \sigma)=\Psi^{\prime}(\sigma)(\Psi(t)-\Psi(\sigma))^{\mu-1} .
$$

Lemma 1. [19] Let $\mu>0, \nu>0$ and $\delta>0$. Then:

(i) $\mathbf{I}_{a^{+}}^{\mu ; \Psi} \mathbf{I}_{a^{+}}^{\nu ; \Psi} h(t)=\mathbf{I}_{a^{+}}^{\mu+\nu ; \Psi} h(t)$;

(ii) If $h(t)=(\Psi(t)-\Psi(a))^{\delta-1}$, then $\quad \mathbf{I}_{a^{+}}^{\mu ;} h(t)=\frac{\Gamma(\delta)}{\Gamma(\mu+\delta)}(\Psi(t)-\Psi(a))^{\mu+\delta-1}$.

Definition 2. [27] The $\Psi$-Hilfer fractional derivative of function $h$ of order $\mu,(0<\mu<1)$ and of type $0 \leq \nu \leq 1$, is defined by

$$
{ }^{H} \mathbf{D}_{a^{+}}^{\mu, \nu ; \Psi} h(t)=\mathbf{I}_{a^{+}}^{\nu(1-\mu) ; \Psi}\left(\frac{1}{\Psi^{\prime}(t)} \frac{d}{d t}\right) \mathbf{I}_{a^{+}}^{(1-\nu)(1-\mu) ; \Psi} h(t) .
$$

Theorem 1. [27] If $h \in C^{1}(\mathcal{J}), 0<\mu<1$ and $0 \leq \nu \leq 1$, then

(i) $\mathbf{I}_{a^{+}}^{\mu ; \Psi}{ }^{H} \mathbf{D}_{a^{+}}^{\mu, \nu ; \Psi} h(t)=h(t)-\Omega_{\Psi}^{\varrho}(t, a) \mathbf{I}_{a^{+}}^{(1-\nu)(1-\mu) ; \Psi} h(a)$,

$$
\Omega_{\Psi}^{\varrho}(t, a)=\frac{(\Psi(t)-\Psi(a))^{\varrho-1}}{\Gamma(\varrho)} ;
$$

(ii) ${ }^{H} \mathbf{D}_{a^{+}}^{\mu, \nu ; \Psi} \mathbf{I}_{a^{+}}^{\mu ; \Psi} h(t)=h(t)$.

Consider the weighted space [27] defined by

$$
C_{1-\varrho ; \Psi}(\mathcal{J})=\left\{u:(a, T] \rightarrow \mathbb{R}:(\Psi(t)-\Psi(a))^{1-\varrho} u(t) \in C(\mathcal{J})\right\}, 0<\varrho \leq 1 .
$$

Define the weighted space of piecewise continuous functions as

$$
\begin{aligned}
& \mathcal{P C}_{1-\varrho ; \Psi}(\mathcal{J}, \mathbb{R})=\left\{u:(a, T] \rightarrow \mathbb{R}: u \in C_{1-\varrho ; \Psi}\left(\left(t_{k}, t_{k+1}\right], \mathbb{R}\right), k=0,1,2, \ldots, m,\right. \\
& \quad \mathbf{I}_{a^{+}}^{1-\varrho ; \Psi} u\left(t_{k}^{+}\right), \mathbf{I}_{a^{+}}^{1-\varrho ; \Psi} u\left(t_{k}^{-}\right) \text {exists and } \mathbf{I}_{a^{+}}^{1-\varrho ; \Psi} u\left(t_{k}^{-}\right)=\mathbf{I}_{a^{+}}^{1-\varrho ; \Psi} u\left(t_{k}\right) \\
& \quad \text { for } k=1,2, \ldots, m\}
\end{aligned}
$$


Clearly, $\mathcal{P C}_{1-\varrho ; \Psi}(\mathcal{J}, \mathbb{R})$ is a Banach space with the norm

$$
\|u\|_{\mathcal{P C}_{1-\varrho ; \Psi}(\mathcal{J}, \mathbb{R})}=\sup _{t \in \mathcal{J}}\left|(\Psi(t)-\Psi(a))^{1-\varrho} u(t)\right| .
$$

Note that for $\varrho=1$, we get $\mathcal{P} \mathcal{C}_{0 ; \Psi}(\mathcal{J}, \mathbb{R})=P C(\mathcal{J}, \mathbb{R})$ a particular case of the space $\mathcal{P} \mathcal{C}_{1-\varrho ; \Psi}(\mathcal{J}, \mathbb{R})$, whose details are given in $[6,12,35]$.

With suitable modification, the PC-type Arzela-Ascoli Theorem [7,38] can be extended to the weighted space $\mathcal{P} \mathcal{C}_{1-\varrho ; \Psi}(\mathcal{J}, \mathcal{X})$.

Theorem $2\left[\mathcal{P C}_{1-\varrho ; \Psi}\right.$ type Arzela-Ascoli Theorem]. Let $\mathcal{X}$ be a Banach space and $\mathcal{W}_{1-\varrho ; \Psi} \subset \mathcal{P C}_{1-\varrho ; \Psi}(\mathcal{J}, \mathcal{X})$. If the following conditions are satisfied:

(a) $\mathcal{W}_{1-\varrho ; \Psi}$ is uniformly bounded subset of $\mathcal{P} \mathcal{C}_{1-\varrho ; \Psi}(\mathcal{J}, \mathcal{X})$;

(b) $\mathcal{W}_{1-\varrho ; \Psi}$ is equicontinuous in $\left(t_{k}, t_{k+1}\right), k=0,1,2, \ldots, m$, where $t_{0}=$ $a, t_{m+1}=T$

(c) $\mathcal{W}_{1-\varrho ; \Psi}(t)=\left\{u(t): u \in \mathcal{W}_{1-\varrho ; \Psi}, t \in \mathcal{J} \backslash\left\{t_{1}, \ldots, t_{m}\right\}, \mathcal{W}_{1-\varrho ; \Psi}\left(t_{k}^{+}\right)=\right.$ $\left\{u\left(t_{k}^{+}\right): u \in \mathcal{W}_{1-\varrho ; \Psi}\right\}$ and $\mathcal{W}_{1-\varrho ; \Psi}\left(t_{k}^{-}\right)=\left\{u\left(t_{k}^{-}\right): u \in \mathcal{W}_{1-\varrho ; \Psi}\right\}$ are relatively compact subsets of $X$,

then $\mathcal{W}_{1-\varrho ; \Psi}$ is a relatively compact subset of $\mathcal{P} \mathcal{C}_{1-\varrho ; \Psi}(\mathcal{J}, X)$.

Proof. Let $\mathcal{W}_{1-\varrho ; \Psi} \subset \mathcal{P C}_{1-\varrho ; \Psi}(\mathcal{J}, \mathcal{X})$ satisfy the conditions (a) to (c). Let $\left\{z_{n}\right\}$ be any sequence in $\mathcal{W}_{1-\varrho ; \Psi}$. Define $x_{n}(t)=(\Psi(t)-\Psi(a))^{1-\varrho} z_{n}(t), \forall n$. Then sequence $\left\{x_{n}\right\} \subset \mathcal{W} \subset P C(\mathcal{J}, \mathcal{X})$, where $\mathcal{W}$ satisfy the conditions of Theorem 2.1 of [38]. Proceeding as in the proof of Theorem 2.1 of [38], there exist $x \in P C(\mathcal{J}, \mathcal{X})$ such that $x_{n} \rightarrow x$ in $P C(\mathcal{J}, \mathcal{X})$ which in turn gives $z_{n} \rightarrow z$ in $\mathcal{P} \mathcal{C}_{1-\varrho ; \Psi}(\mathcal{J}, \mathcal{X})$. This proves $\mathcal{W}_{1-\varrho ; \Psi}$ is a relatively compact subset of $\mathcal{P C}_{1-\varrho ; \Psi}(\mathcal{J}, \mathcal{X})$.

Theorem 3 [Krasnoselskii, [39]]. Let $\mathcal{M}$ be a closed, convex, and nonempty subset of a Banach space $\mathcal{X}$, and $A, B$ the operators such that

1. $\mathcal{A} x+\mathcal{B} y \in \mathcal{M}$ whenever $x, y \in \mathcal{M}$;

2. $\mathcal{A}$ is compact and continuous; $3 . \mathcal{B}$ is a contraction mapping.

Then there exists $z \in \mathcal{M}$ such that $z=\mathcal{A} z+\mathcal{B} z$.

\section{Representation formula for the solution}

The following lemma play an important role in building an equivalent fractional integral equation of the impulsive $\Psi$-HFDE (1.2)-(1.4).

Lemma 2. Let $0<\mu<1$ and $0 \leq \nu \leq 1, \varrho=\mu+\nu-\mu \nu$ and $h: \mathcal{J} \rightarrow \mathbb{R}$ be continuous. Then for any $b \in \mathcal{J}$ a function $u \in C_{1-\varrho, \Psi}(\mathcal{J}, \mathbb{R})$ defined by

$$
u(t)=\Omega_{\Psi}^{\varrho}(t, a)\left\{\mathbf{I}_{a^{+}}^{1-\varrho ; \Psi} u(b)-\left.\mathbf{I}_{a^{+}}^{1-\varrho+\mu ; \Psi} h(t)\right|_{t=b}\right\}+\mathbf{I}_{a^{+}}^{\mu ; \Psi} h(t)
$$

is the solution of the $\Psi$-Hilfer fractional differential equation

$$
{ }^{H} \mathbf{D}_{a^{+}}^{\mu, \nu ; \Psi} u(t)=h(t), t \in \mathcal{J} .
$$


Proof. Applying ${ }^{H} \mathbf{D}_{a^{+}}^{\mu, \nu ; \Psi}$ on both sides of the equation (3.1), we get

$$
\begin{gathered}
{ }^{H} \mathbf{D}_{a^{+}}^{\mu, \nu ; \Psi} u(t)=\left\{\mathbf{I}_{a^{+}}^{1-\varrho ; \Psi} u(b)-\left.\mathbf{I}_{a^{+}}^{1-\varrho+\mu ; \Psi} h(t)\right|_{t=b}\right\}{ }^{H} \mathbf{D}_{a^{+}}^{\mu, \nu ; \Psi} \Omega_{\Psi}^{\varrho}(t, a) \\
+{ }^{H} \mathbf{D}_{a^{+}}^{\mu, \nu ; \Psi} \mathbf{I}_{a^{+}}^{\mu ; \Psi} h(t), t \in \mathcal{J} .
\end{gathered}
$$

Using the result ( [33], Page 10),

$$
{ }^{H} \mathbf{D}_{a^{+}}^{\mu, \nu ;}(\Psi(t)-\Psi(a))^{\varrho-1}=0,0<\varrho<1,
$$

and using the Theorem 1 , we get ${ }^{H} \mathbf{D}_{a^{+}}^{\mu, \nu ; \Psi} u(t)=h(t), t \in \mathcal{J}$. This completes the proof of the Lemma.

In the next result, utilizing the Lemma 2, we obtain the equivalent fractional integral of the problem (1.2)-(1.4).

Lemma 3. Let $h: \mathcal{J} \rightarrow \mathbb{R}$ be a continuous function. Then a function $u \in$ $\mathcal{P C}_{1-\varrho ; \Psi}(\mathcal{J}, \mathbb{R})$ is a solution of impulsive $\Psi-H F D E$

$$
\begin{aligned}
{ }^{H} \mathbf{D}_{a^{+}}^{\mu, \nu ; \Psi} u(t) & =h(t), t \in \mathcal{J} \backslash\left\{t_{1}, t_{2}, \ldots, t_{m}\right\}, \\
\Delta \mathbf{I}_{a^{+}}^{1-\varrho ; \Psi} u\left(t_{k}\right) & =\zeta_{k} \in \mathbb{R}, \quad k=1,2,3, \ldots, m, \\
\mathbf{I}_{a^{+}}^{1-\varrho ; \Psi} u(a) & =\delta \in \mathbb{R},
\end{aligned}
$$

if and only if $u$ is a solution of the following fractional integral equation

$$
u(t)=\left\{\begin{array}{l}
\Omega_{\Psi}^{\varrho}(t, a) \delta+\mathbf{I}_{a^{+}}^{\mu ;} h(t), t \in\left[a, t_{1}\right], \\
\Omega_{\Psi}^{\varrho}(t, a)\left(\delta+\sum_{i=1}^{k} \zeta_{i}\right)+\mathbf{I}_{a^{+}}^{\mu ; \Psi} h(t), t \in\left(t_{k}, t_{k+1}\right], k=1, \ldots, m .
\end{array}\right.
$$

Proof. Assume that $u \in \mathcal{P C}_{1-\varrho ; \Psi}(\mathcal{J}, \mathbb{R})$ satisfies the impulsive $\Psi$-HFDE (3.3)-(3.5). If $t \in\left[a, t_{1}\right]$ then

$$
\left\{\begin{array}{l}
{ }^{H} \mathbf{D}_{a}^{\mu, \nu ; \Psi} u(t)=h(t) \\
\mathbf{I}_{a^{+}}^{1-\varrho ; \Psi} u(a)=\delta
\end{array}\right.
$$

Then the problem (3.7) is equivalent to the following fractional integral [33]

$$
u(t)=\Omega_{\Psi}^{\varrho}(t, a) \delta+\mathbf{I}_{a^{+}}^{\mu ; \Psi} h(t), \quad t \in\left[a, t_{1}\right] .
$$

Now, if $t \in\left(t_{1}, t_{2}\right]$ then

$$
{ }^{H} \mathbf{D}_{a^{+}}^{\mu, \nu ; \Psi} u(t)=h(t), t \in\left(t_{1}, t_{2}\right] \text { with } \mathbf{I}_{a^{+}}^{1-\varrho ; \Psi} u\left(t_{1}^{+}\right)-\mathbf{I}_{a^{+}}^{1-\varrho ; \Psi} u\left(t_{1}^{-}\right)=\zeta_{1} .
$$

By Lemma 2, we have

$$
\begin{aligned}
& u(t)=\Omega_{\Psi}^{\varrho}(t, a)\left\{\mathbf{I}_{a^{+}}^{1-\varrho ; \Psi} u\left(t_{1}^{+}\right)-\left.\mathbf{I}_{a^{+}}^{1-\varrho+\mu ; \Psi} h(t)\right|_{t=t_{1}}\right\}+\mathbf{I}_{a^{+}}^{\mu ; \Psi} h(t) \\
& =\Omega_{\Psi}^{\varrho}(t, a)\left\{\mathbf{I}_{a^{+}}^{1-\varrho ; \Psi} u\left(t_{1}^{-}\right)+\zeta_{1}-\left.\mathbf{I}_{a^{+}}^{1-\varrho+\mu ; \Psi} h(t)\right|_{t=t_{1}}\right\}+\mathbf{I}_{a^{+}}^{\mu ; \Psi} h(t), t \in\left(t_{1}, t_{2}\right] .
\end{aligned}
$$


Now, from (3.8), we have $\mathbf{I}_{a^{+}}^{1-\varrho ; \Psi} u(t)=\delta+\mathbf{I}_{a^{+}}^{1-\varrho+\mu ; \Psi} h(t)$. This gives

$$
\mathbf{I}_{a^{+}}^{1-\varrho ; \Psi} u\left(t_{1}^{-}\right)-\left.\mathbf{I}_{a^{+}}^{1-\varrho+\mu ; \Psi} h(t)\right|_{t=t_{1}}=\delta .
$$

Using (3.10) in (3.9), we obtain

$$
u(t)=\Omega_{\Psi}^{\varrho}(t, a)\left(\delta+\zeta_{1}\right)+\mathbf{I}_{a^{+}}^{\mu ; \Psi} h(t), t \in\left(t_{1}, t_{2}\right] .
$$

Next, if $t \in\left(t_{2}, t_{3}\right]$ then

$$
{ }^{H} \mathbf{D}_{a^{+}}^{\mu, \nu ; \Psi} u(t)=h(t), t \in\left(t_{2}, t_{3}\right] \text { with } \mathbf{I}_{a^{+}}^{1-\varrho ; \Psi} u\left(t_{2}^{+}\right)-\mathbf{I}_{a^{+}}^{1-\varrho ; \Psi} u\left(t_{2}^{-}\right)=\zeta_{2} .
$$

Again by Lemma 2, we have

$$
\begin{aligned}
& u(t)=\Omega_{\Psi}^{\varrho}(t, a)\left\{\mathbf{I}_{a^{+}}^{1-\varrho ; \Psi} u\left(t_{2}^{+}\right)-\left.\mathbf{I}_{a^{+}}^{1-\varrho+\mu ; \Psi} h(t)\right|_{t=t_{2}}\right\}+\mathbf{I}_{a^{+}}^{\mu ; \Psi} h(t)=\Omega_{\Psi}^{\varrho}(t, a) \\
& \times\left\{\mathbf{I}_{a^{+}}^{1-\varrho ; \Psi} u\left(t_{2}^{-}\right)+\zeta_{2}-\left.\mathbf{I}_{a^{+}}^{1-\varrho+\mu ; \Psi} h(t)\right|_{t=t_{2}}\right\}+\mathbf{I}_{a^{+}}^{\mu ; \Psi} h(t), t \in\left(t_{2}, t_{3}\right] .
\end{aligned}
$$

From (3.11), we have $\mathbf{I}_{a^{+}}^{1-\varrho ; \Psi} u(t)=\left(\delta+\zeta_{1}\right)+\mathbf{I}_{a^{+}}^{1-\varrho+\mu ; \Psi} h(t)$, which gives

$$
\mathbf{I}_{a^{+}}^{1-\varrho ; \Psi} u\left(t_{2}^{-}\right)-\left.\mathbf{I}_{a^{+}}^{1-\varrho+\mu ; \Psi} h(t)\right|_{t=t_{2}}=\delta+\zeta_{1}
$$

Using (3.13) in (3.12), we get

$$
u(t)=\Omega_{\Psi}^{\varrho}(t, a)\left(\delta+\zeta_{1}+\zeta_{2}\right)+\mathbf{I}_{a^{+}}^{\mu ; \Psi} h(t), t \in\left(t_{2}, t_{3}\right] .
$$

Continuing the above process, we obtain

$$
u(t)=\Omega_{\Psi}^{\varrho}(t, a)\left(\delta+\sum_{i=1}^{k} \zeta_{i}\right)+\mathbf{I}_{a^{+}}^{\mu ; \Psi} h(t), \quad t \in\left(t_{k}, t_{k+1}\right], k=1,2, \ldots, m .
$$

Conversely, let $u \in \mathcal{P C}_{1-\varrho ; \Psi}(\mathcal{J}, \mathbb{R})$ satisfies the fractional integral equation (3.6). Then, for $t \in\left[a, t_{1}\right]$, we have $u(t)=\Omega_{\Psi}^{\varrho}(t, a) \delta+\mathbf{I}_{a^{+}}^{\mu ; \Psi} h(t)$. Applying the $\Psi$-Hilfer fractional derivative operator ${ }^{H} D_{a^{+}}^{\mu, \nu ; \Psi}$ on both sides, we get

$$
{ }^{H} \mathbf{D}_{a^{+}}^{\mu, \nu ; \Psi} u(t)=\delta{ }^{H} \mathbf{D}_{a^{+}}^{\mu, \nu ; \Psi} \Omega_{\Psi}^{\varrho}(t, a)+{ }^{H} \mathbf{D}_{a^{+}}^{\mu, \nu ; \Psi} \mathbf{I}_{a^{+}}^{\mu ; \Psi} h(t)
$$

Utilizing (3.2) and Theorem $1,{ }^{H} \mathbf{D}_{a^{+}}^{\mu, \nu ; \Psi} u(t)=h(t), t \in\left[a, t_{1}\right]$. Now, for $t \in$ $\left(t_{k}, t_{k+1}\right],(k=1,2, \ldots, m)$, we have

$$
u(t)=\Omega_{\Psi}^{\varrho}(t, a)\left(\delta+\sum_{i=1}^{k} \zeta_{i}\right)+\mathbf{I}_{a^{+}}^{\mu ; \Psi} h(t), \quad t \in\left(t_{k}, t_{k+1}\right], k=1,2, \ldots, m .
$$

Applying the operator ${ }^{H} \mathbf{D}_{a^{+}}^{\mu, \nu ;}(\cdot)$ on both sides and using (3.2) and the Theorem 1 , we obtain

$$
{ }^{H} \mathbf{D}_{a^{+}}^{\mu, \nu} u(t)=\left\{\delta+\sum_{i=1}^{k} \zeta_{i}\right\}^{H} \mathbf{D}_{a^{+}}^{\mu, \nu ; \Psi} \Omega_{\Psi}^{\varrho}(t, a)+{ }^{H} \mathbf{D}_{a^{+}}^{\mu, \nu ; \Psi} \mathbf{I}_{a^{+}}^{\mu ; \Psi} h(t)=h(t)
$$


We have proved that $u$ satisfies (3.3). Next, we prove that $u$ also satisfy the conditions (3.4) and (3.5). Applying the $\Psi$-RL fractional operator $\mathbf{I}_{a^{+}}^{1-\varrho ; \Psi}(\cdot)$ on both sides of $(3.8)$, we get

$$
\mathbf{I}_{a^{+}}^{1-\varrho ; \Psi} u(t)=\delta \mathbf{I}_{a^{+}}^{1-\varrho ; \Psi} \Omega_{\Psi}^{\varrho}(t, a)+\mathbf{I}_{a^{+}}^{1-\varrho ; \Psi} \mathbf{I}_{a^{+}}^{\mu ; \Psi} h(t)=\delta+\mathbf{I}_{a^{+}}^{1-\varrho+\mu ; \Psi} h(t),
$$

and from which we obtain $\mathbf{I}_{a^{+}}^{1-\varrho ; \Psi} u(a)=\delta$, which is the condition (3.5). Further, from Equation (3.6) for $t \in\left(t_{k}, t_{k+1}\right]$, we have

$$
\begin{aligned}
\mathbf{I}_{a^{+}}^{1-\varrho ; \Psi} u(t) & =\left\{\delta+\sum_{i=1}^{k} \zeta_{i}\right\} \mathbf{I}_{a^{+}}^{1-\varrho ; \Psi} \Omega_{\Psi}^{\varrho}(t, a)+\mathbf{I}_{a^{+}}^{1-\varrho ; \Psi} \mathbf{I}_{a^{+}}^{\mu ; \Psi} h(t) \\
& =\delta+\sum_{i=1}^{k} \zeta_{i}+\mathbf{I}_{a^{+}}^{1-\varrho+\mu ; \Psi} h(t)
\end{aligned}
$$

and for $t \in\left(t_{k-1}, t_{k}\right]$ we have

$$
\begin{aligned}
\mathbf{I}_{a^{+}}^{1-\varrho ; \Psi} u(t) & =\left\{\delta+\sum_{i=1}^{k-1} \zeta_{i}\right\} \mathbf{I}_{a^{+}}^{1-\varrho ; \Psi} \Omega_{\Psi}^{\varrho}(t, a)+\mathbf{I}_{a^{+}}^{1-\varrho ; \Psi} \mathbf{I}_{a^{+}}^{\mu ; \Psi} h(t) \\
& =\delta+\sum_{i=1}^{k-1} \zeta_{i}+\mathbf{I}_{a^{+}}^{1-\varrho+\mu ; \Psi} h(t) .
\end{aligned}
$$

Therefore, from (3.14) to (3.15), we obtain

$$
\mathbf{I}_{a^{+}}^{1-\varrho ; \Psi} u\left(t_{k}^{+}\right)-\mathbf{I}_{a^{+}}^{1-\varrho ; \Psi} u\left(t_{k}^{-}\right)=\sum_{i=1}^{k} \zeta_{i}-\sum_{i=1}^{k-1} \zeta_{i}=\zeta_{k},
$$

which condition (3.4). We have proved that $u$ satisfies the impulsive $\Psi$-HFDE (3.3)-(3.5). This completes the proof.

\section{Existence and uniqueness results}

Theorem 4. (Existence) Assume that the function $f:(a, T] \times \mathbb{R} \rightarrow \mathbb{R}$ is continuous and satisfies the conditions:

$\left(A_{1}\right) f(\cdot, u(\cdot)) \in \mathcal{P C}_{1-\varrho ; \Psi}(\mathcal{J}, \mathbb{R})$ for any $u \in \mathcal{P} \mathcal{C}_{1-\varrho ; \Psi}(\mathcal{J}, \mathbb{R})$,

$\left(A_{2}\right)$ there exists a constant $0<L \leq \Gamma(\mu+\varrho) /\left(2 \Gamma(\varrho)(\Psi(T)-\Psi(a))^{\mu}\right)$ satisfying

$$
|f(t, u)-f(t, v)| \leq L|u-v|, t \in \mathcal{J}, u, v \in \mathbb{R} .
$$

Then, the impulsive $\Psi-H F D E$ (1.2)-(1.4) has at least one solution in $\mathcal{P C}_{1-\varrho ; \Psi}(\mathcal{J}, \mathbb{R})$.

Proof. In the view of Lemma 3, the equivalent fractional integral equation of the impulsive $\Psi$-HFDE (1.2)-(1.4) is given by

$$
u(t)=\Omega_{\Psi}^{\varrho}(t, a)\left(\delta+\sum_{a<t_{k}<t} \zeta_{k}\right)+\mathbf{I}_{a^{+}}^{\mu ; \Psi} f(t, u(t)), t \in \mathcal{J} .
$$


Consider the set

$$
\mathcal{B}_{r}=\left\{u \in \mathcal{P C}_{1-\varrho ; \Psi}(\mathcal{J}, \mathbb{R}): \mathbf{I}_{a^{+}}^{1-\varrho ; \Psi} u(a)=\delta,\|u\|_{\mathcal{P C}_{1-\varrho ; \Psi}(\mathcal{J}, \mathbb{R})} \leq r\right\},
$$

where

$$
\mathcal{M}=\sup _{\sigma \in \mathcal{J}}|f(\sigma, 0)|, r \geq 2\left(\frac{1}{\Gamma(\varrho)}\left\{|\delta|+\sum_{i=1}^{m}\left|\zeta_{i}\right|\right\}+\frac{\mathcal{M}(\Psi(T)-\Psi(a))^{1-\varrho+\mu}}{\Gamma(\mu+1)}\right) .
$$

We define the operators $\mathcal{P}$ and $\mathcal{Q}$ on $\mathcal{B}_{r}$ by

$$
\mathcal{P} u(t)=\Omega_{\Psi}^{\varrho}(t, a)\left(\delta+\sum_{a<t_{k}<t} \zeta_{k}\right), \mathcal{Q} u(t)=\mathbf{I}_{a^{+}}^{\mu ; \Psi} f(t, u(t)), t \in \mathcal{J} .
$$

Then the fractional integral equation (4.1) can be written as operator equation

$$
u=\mathcal{P} u+\mathcal{Q} u, u \in \mathcal{P C}_{1-\varrho ; \Psi}(\mathcal{J}, \mathbb{R}) .
$$

Step 1: We prove that $\mathcal{P} u+\mathcal{Q} v \in \mathcal{B}_{r}$ for any $u, v \in \mathcal{B}_{r}$.

Let any $u, v \in \mathcal{B}_{r}$. Then using $\left(A_{1}\right)$, for any $t \in \mathcal{J}$, we have

$$
\begin{aligned}
& \left|(\Psi(t)-\Psi(a))^{1-\varrho}(\mathcal{P} u(t)+\mathcal{Q} v(t))\right| \\
& =\left|(\Psi(t)-\Psi(a))^{1-\varrho}\left\{\Omega_{\Psi}^{\varrho}(t, a)\left(\delta+\sum_{a<t_{k}<t} \zeta_{k}\right)+\mathbf{I}_{a^{+}}^{\mu ; \Psi} f(t, v(t))\right\}\right| \\
& \leq \frac{1}{\Gamma(\varrho)}\left(|\delta|+\sum_{k=1}^{m}\left|\zeta_{k}\right|\right)+\frac{(\Psi(t)-\Psi(a))^{1-\varrho}}{\Gamma(\mu)} \int_{a}^{t} \mathcal{L}_{\Psi}^{\mu}(t, \sigma)|f(\sigma, v(\sigma))| d \sigma \\
& \leq \frac{1}{\Gamma(\varrho)}\left(|\delta|+\sum_{k=1}^{m}\left|\zeta_{k}\right|\right)+\frac{(\Psi(t)-\Psi(a))^{1-\varrho}}{\Gamma(\mu)} \int_{a}^{t} \mathcal{L}_{\Psi}^{\mu}(t, \sigma)|f(\sigma, v(\sigma))-f(\sigma, 0)| d \sigma \\
& +\frac{(\Psi(t)-\Psi(a))^{1-\varrho}}{\Gamma(\mu)} \int_{a}^{t} \mathcal{L}_{\Psi}^{\mu}(t, \sigma)|f(\sigma, 0)| d \sigma \\
& \leq \frac{1}{\Gamma(\varrho)}\left(|\delta|+\sum_{k=1}^{m}\left|\zeta_{k}\right|\right)+\frac{L(\Psi(t)-\Psi(a))^{1-\varrho}}{\Gamma(\mu)} \int_{a}^{t} \mathcal{L}_{\Psi}^{\mu}(t, \sigma)|v(\sigma)| d \sigma \\
& +\frac{\mathcal{M}(\Psi(t)-\Psi(a))^{1-\varrho}}{\Gamma(\mu)} \int_{a}^{t} \mathcal{L}_{\Psi}^{\mu}(t, \sigma) d \sigma=\frac{1}{\Gamma(\varrho)}\left(|\delta|+\sum_{k=1}^{m}\left|\zeta_{k}\right|\right) \\
& +\frac{L(\Psi(t)-\Psi(a))^{1-\varrho}}{\Gamma(\mu)} \int_{a}^{t} \mathcal{L}_{\Psi}^{\mu}(t, \sigma)(\Psi(\sigma)-\Psi(a))^{\varrho-1}\left|(\Psi(\sigma)-\Psi(a))^{1-\varrho} v(\sigma)\right| d \sigma \\
& +\frac{\mathcal{M}(\Psi(t)-\Psi(a))^{1-\varrho}}{\Gamma(\mu)} \frac{(\Psi(t)-\Psi(a))^{\mu}}{\mu} \leq \frac{1}{\Gamma(\varrho)}\left(|\delta|+\sum_{k=1}^{m}\left|\zeta_{k}\right|\right)+L(\Psi(t)-\Psi(a))^{1-\varrho} \\
& \times\|v\|_{\mathcal{P C}_{1-\varrho ; \Psi}(\mathcal{J}, \mathbb{R})} \mathbf{I}_{a^{+}}^{\mu ; \Psi}(\Psi(t)-\Psi(a))^{\varrho-1}+\frac{\mathcal{M}(\Psi(t)-\Psi(a))^{1-\varrho+\mu}}{\Gamma(\mu+1)} \\
& \leq \frac{1}{\Gamma(\varrho)}\left(|\delta|+\sum_{k=1}^{m}\left|\zeta_{k}\right|\right)+\frac{L \Gamma(\varrho)}{\Gamma(\mu+\varrho)}(\Psi(t)-\Psi(a))^{\mu}\|v\|_{\mathcal{P C} \mathcal{C}_{1-\varrho} \Psi}(\mathcal{J}, \mathbb{R})
\end{aligned}
$$




$$
\begin{gathered}
+\frac{\mathcal{M}(\Psi(t)-\Psi(a))^{1-\varrho+\mu}}{\Gamma(\mu+1)} \leq \frac{1}{\Gamma(\varrho)}\left(|\delta|+\sum_{k=1}^{m}\left|\zeta_{k}\right|\right) \\
+\frac{L \Gamma(\varrho)}{\Gamma(\mu+\varrho)}(\Psi(T)-\Psi(a))^{\mu} r+\frac{\mathcal{M}(\Psi(T)-\Psi(a))^{1-\varrho+\mu}}{\Gamma(\mu+1)} .
\end{gathered}
$$

Since

$$
\begin{aligned}
& r \geq 2\left(\frac{1}{\Gamma(\varrho)}\left\{|\delta|+\sum_{i=1}^{m}\left|\zeta_{i}\right|\right\}+\frac{\mathcal{M}(\Psi(T)-\Psi(a))^{1-\varrho+\mu}}{\Gamma(\mu+1)}\right), \\
& L \leq \Gamma(\mu+\varrho) /\left(2 \Gamma(\varrho)(\Psi(T)-\Psi(a))^{\mu}\right),
\end{aligned}
$$

we have $\left|(\Psi(t)-\Psi(a))^{1-\varrho}(\mathcal{P} u(t)+\mathcal{Q} v(t))\right| \leq r, t \in \mathcal{J}$. Therefore $\|(\mathcal{P} u+\mathcal{Q} v)\|_{\mathcal{P C}_{1-\varrho ; \Psi}(\mathcal{J}, \mathbb{R})} \leq r$. From definition of the operator $\mathcal{P}$ and $\mathcal{Q}$, one can verify that $\mathbf{I}_{a^{+}}^{1-\varrho ; \Psi}(\mathcal{P} u+\mathcal{Q} v)(a)=\delta$. We have proved that, $\mathcal{P} u+\mathcal{Q} v \in \mathcal{B}_{r}$. Step 2 : Clearly $\mathcal{P}$ is a contraction with the contraction constant zero.

Step 3: $\mathcal{Q}$ is compact and continuous. The continuity of $\mathcal{Q}$ follows from the continuity of $f$. Next we prove that $\mathcal{Q}$ is uniformly bounded on $\mathcal{B}_{r}$. Let any $u \in \mathcal{B}_{r}$. Then by $\left(A_{2}\right)$, for any $t \in \mathcal{J}$, we have

$$
\begin{aligned}
& \left|(\Psi(t)-\Psi(a))^{1-\varrho} \mathcal{Q} u(t)\right|=\left|(\Psi(t)-\Psi(a))^{1-\varrho} \mathbf{I}_{a^{+}}^{\mu ; \Psi} f(t, u(t))\right| \\
& \leq \frac{(\Psi(t)-\Psi(a))^{1-\varrho}}{\Gamma(\mu)} \int_{a}^{t} \mathcal{L}_{\Psi}^{\mu}(t, \sigma)|f(\sigma, u(\sigma))| d \sigma \leq \frac{(\Psi(t)-\Psi(a))^{1-\varrho}}{\Gamma(\mu)} \\
& \times \int_{a}^{t} \mathcal{L}_{\Psi}^{\mu}(t, \sigma)|f(\sigma, u(\sigma))-f(\sigma, 0)| d \sigma+\frac{(\Psi(t)-\Psi(a))^{1-\varrho}}{\Gamma(\mu)} \int_{a}^{t} \mathcal{L}_{\Psi}^{\mu}(t, \sigma)|f(\sigma, 0)| d \sigma \\
& \leq \frac{L(\Psi(t)-\Psi(a))^{1-\varrho}}{\Gamma(\mu)} \int_{a}^{t} \mathcal{L}_{\Psi}^{\mu}(t, \sigma)|u(\sigma)| d \sigma+\frac{M(\Psi(t)-\Psi(a))^{1-\varrho}}{\Gamma(\mu)} \int_{a}^{t} \mathcal{L}_{\Psi}^{\mu}(t, \sigma) d \sigma \\
& \leq \frac{L \Gamma(\varrho)}{\Gamma(\mu+\varrho)}(\Psi(t)-\Psi(a))^{\mu}\|u\|_{\mathcal{P C}_{1-\varrho ; \Psi}(\mathcal{J}, \mathbb{R})}+\frac{\mathcal{M}(\Psi(t)-\Psi(a))^{1-\varrho+\mu}}{\Gamma(\mu+1)} \\
& \leq \frac{L \Gamma(\varrho)}{\Gamma(\mu+\varrho)}(\Psi(T)-\Psi(a))^{\mu} r+\frac{\mathcal{M}(\Psi(T)-\Psi(a))^{1-\varrho+\mu}}{\Gamma(\mu+1)} .
\end{aligned}
$$

Therefore

$$
\|\mathcal{Q} u\|_{\mathcal{P C}_{1-\varrho ; \Psi}(\mathcal{J}, \mathbb{R})} \leq \frac{L \Gamma(\varrho)}{\Gamma(\mu+\varrho)}(\Psi(T)-\Psi(a))^{\mu} r+\frac{\mathcal{M}(\Psi(T)-\Psi(a))^{1-\varrho+\mu}}{\Gamma(\mu+1)} .
$$

This proves $\mathcal{Q}$ is uniformly bounded on $\mathcal{B}_{r}$. Next, we show that $\mathcal{Q} \mathcal{B}_{r}$ is equicontinuous. Let any $u \in \mathcal{B}_{r}$ and $t_{1}, t_{2} \in\left(t_{k}, t_{k+1}\right]$ for some $k,(k=0,1, \ldots, m)$ with $t_{1}<t_{2}$. Then,

$$
\begin{aligned}
& \left|\mathcal{Q} u\left(t_{2}\right)-\mathcal{Q} u\left(t_{1}\right)\right|=\left|\left(\left.\mathbf{I}_{a^{+}}^{\mu ; \Psi} f(t, u(t))\right|_{t=t_{2}}\right)-\left(\left.\mathbf{I}_{a^{+}}^{\mu ; \Psi} f(t, u(t))\right|_{t=t_{1}}\right)\right| \\
& \leq \frac{1}{\Gamma(\mu)} \int_{a}^{t_{2}} \mathcal{L}_{\Psi}^{\mu}\left(t_{2}, \sigma\right)|f(\sigma, u(\sigma))| d \sigma-\frac{1}{\Gamma(\mu)} \int_{a}^{t_{1}} \mathcal{L}_{\Psi}^{\mu}\left(t_{1}, \sigma\right)|f(\sigma, u(\sigma))| d \sigma \\
& =\frac{1}{\Gamma(\mu)} \int_{a}^{t_{2}} \mathcal{L}_{\Psi}^{\mu}\left(t_{2}, \sigma\right)(\Psi(\sigma)-\Psi(a))^{\varrho-1}\left|(\Psi(\sigma)-\Psi(a))^{1-\varrho} f(\sigma, u(\sigma))\right| d \sigma
\end{aligned}
$$




$$
\begin{aligned}
& -\frac{1}{\Gamma(\mu)} \int_{a}^{t_{1}} \mathcal{L}_{\Psi}^{\mu}\left(t_{1}, \sigma\right)(\Psi(\sigma)-\Psi(a))^{\varrho-1}\left|(\Psi(\sigma)-\Psi(a))^{1-\varrho} f(\sigma, u(\sigma))\right| d \sigma \\
& \leq\left\{\left.\mathbf{I}_{a^{+}}^{\mu ; \Psi}(\Psi(t)-\Psi(a))^{\varrho-1}\right|_{t=t_{2}}-\left.\mathbf{I}_{a^{+}}^{\mu ; \Psi}(\Psi(t)-\Psi(a))^{\varrho-1}\right|_{t=t_{1}}\right\}\|f\|_{\mathcal{P C}_{1-\varrho ; \Psi}(\mathcal{J}, \mathbb{R})} \\
& =\frac{\Gamma(\varrho)}{\Gamma(\mu+\varrho)}\left\{\left(\Psi\left(t_{2}\right)-\Psi(a)\right)^{1-\varrho+\mu}-\left(\Psi\left(t_{1}\right)-\Psi(a)\right)^{1-\varrho+\mu}\right\}\|f\|_{\mathcal{P} \mathcal{C}_{1-\varrho ; \Psi}(\mathcal{J}, \mathbb{R})} .
\end{aligned}
$$

Note that $\left|\mathcal{Q} u\left(t_{2}\right)-\mathcal{Q} u\left(t_{1}\right)\right| \rightarrow 0$ as $\left|t_{1}-t_{2}\right| \rightarrow 0$. This shows that $\mathcal{Q}$ is equicontinuous on $\left(t_{k}, t_{k+1}\right]$. Therefore $\mathcal{Q}$ is relatively compact on $\mathcal{B}_{r}$. By $\mathcal{P C}_{1-\varrho ; \Psi}$ type Arzela-Ascoli Theorem (Theorem 2) $\mathcal{Q}$ is compact on $\mathcal{B}_{r}$. Since all the assumptions of Krasnoselskii's fixed point theorem (Theorem 3 ) are satisfied, the operator equation $u=\mathcal{P} u+\mathcal{Q} u$ has fixed point $\tilde{u} \in \mathcal{P} \mathcal{C}_{1-\varrho ; \Psi}(\mathcal{J}, \mathbb{R})$, which is the solution of the impulsive $\Psi$-HFDE (1.2)-(1.4).

Theorem 5. (Uniqueness) Assume that the function $f:(a, T] \times \mathbb{R} \rightarrow \mathbb{R}$ is continuous and satisfies the conditions $\left(A_{1}\right)-\left(A_{2}\right)$. Then, impulsive $\Psi-H F D E$ (1.2)-(1.4) has a unique solution in the weighted space $\mathcal{P C}_{1-\varrho ; \Psi}(\mathcal{J}, \mathbb{R})$.

Proof. Consider the set $\mathcal{B}_{r}$ as defined in the Theorem 4 and define the operator $\mathcal{T}$ on $\mathcal{B}_{r}$ by

$$
\mathcal{T} u(t)=\Omega_{\Psi}^{\varrho}(t, a)\left(\delta+\sum_{a<t_{k}<t} \zeta_{k}\right)+\mathbf{I}_{a^{+}}^{\mu ; \Psi} f(t, u(t)), t \in \mathcal{J} .
$$

To prove $u=\mathcal{T} u$ has a fixed point, we show that $\mathcal{T} \mathcal{B}_{r} \subset \mathcal{B}_{r}$. For that take any $u \in \mathcal{B}_{r}$. Then, by $\left(A_{2}\right)$ for any $t \in \mathcal{J}$, we have

$$
\begin{aligned}
& |\mathcal{T} u(t)|=\left|\Omega_{\Psi}^{\varrho}(t, a)\left(\delta+\sum_{a<t_{k}<t} \zeta_{k}\right)+\mathbf{I}_{a^{+}}^{\mu ; \Psi} f(t, u(t))\right| \\
& \leq \Omega_{\Psi}^{\varrho}(t, a)\left(|\delta|+\sum_{k=1}^{m}\left|\zeta_{k}\right|\right)+\frac{1}{\Gamma(\mu)} \int_{a}^{t} \mathcal{L}_{\Psi}^{\mu}(t, \sigma)|f(\sigma, u(\sigma))| d \sigma \\
& \leq \Omega_{\Psi}^{\varrho}(t, a)\left(|\delta|+\sum_{k=1}^{m}\left|\zeta_{k}\right|\right)+\frac{1}{\Gamma(\mu)} \int_{a}^{t} \mathcal{L}_{\Psi}^{\mu}(t, \sigma)|f(\sigma, u(\sigma))-f(\sigma, 0)| d \sigma \\
& +\frac{1}{\Gamma(\mu)} \int_{a}^{t} \mathcal{L}_{\Psi}^{\mu}(t, \sigma)|f(\sigma, 0)| d \sigma \leq \Omega_{\Psi}^{\varrho}(t, a)\left(|\delta|+\sum_{k=1}^{m}\left|\zeta_{k}\right|\right) \\
& +\frac{L \Gamma(\varrho)}{\Gamma(\mu+\varrho)}(\Psi(t)-\Psi(a))^{1-\varrho+\mu}\|u\|_{\mathcal{P C}_{1-\varrho ; \Psi}(\mathcal{J}, \mathbb{R})}+\frac{\mathcal{M}(\Psi(t)-\Psi(a))^{\mu}}{\Gamma(\mu+1)} \\
& \leq \Omega_{\Psi}^{\varrho}(t, a)\left(|\delta|+\sum_{k=1}^{m}\left|\zeta_{k}\right|\right)+\frac{L \Gamma(\varrho)}{\Gamma(\mu+\varrho)}(\Psi(t)-\Psi(a))^{1-\varrho+\mu} r \\
& +\frac{\mathcal{M}(\Psi(t)-\Psi(a))^{\mu}}{\Gamma(\mu+1)}
\end{aligned}
$$

Thus,

$$
\left|(\Psi(t)-\Psi(a))^{1-\varrho} \mathcal{T} u(t)\right| \leq \frac{1}{\Gamma(\varrho)}\left(|\delta|+\sum_{k=1}^{m}\left|\zeta_{k}\right|\right)+\frac{L \Gamma(\varrho)}{\Gamma(\mu+\varrho)}(\Psi(T)-\Psi(a))^{\mu} r
$$




$$
+\frac{\mathcal{M}(\Psi(T)-\Psi(a))^{1-\varrho+\mu}}{\Gamma(\mu+1)}, t \in \mathcal{J} .
$$

From the choices of constants $r$ and $L$, it can be easily verified that

$$
\|\mathcal{T} u\|_{\mathcal{P C}_{1-\varrho ; \Psi}(\mathcal{J}, \mathbb{R})} \leq r .
$$

This proves $\mathcal{T} \mathcal{B}_{r} \subset \mathcal{B}_{r}$. Now, we prove that the operator $\mathcal{T}$ is a contraction on $\mathcal{B}_{r}$. Let any $u, v \in \mathcal{B}_{r}$. Then by assumption $\left(A_{2}\right)$ for any $t \in \mathcal{J}$,

$$
\begin{aligned}
& \left|(\Psi(t)-\Psi(a))^{1-\varrho}(\mathcal{T} u(t)-\mathcal{T} v(t))\right| \\
& =\mid(\Psi(t)-\Psi(a))^{1-\varrho}\left(\left\{\Omega_{\Psi}^{\varrho}(t, a)\left(\delta+\sum_{a<t_{k}<t} \zeta_{k}\right)+\mathbf{I}_{a^{+}}^{\mu ; \Psi} f(t, u(t))\right\}\right. \\
& \left.-\left\{\Omega_{\Psi}^{\varrho}(t, a)\left(\delta+\sum_{a<t_{k}<t} \zeta_{k}\right)+\mathbf{I}_{a^{+}}^{\mu ; \Psi} f(t, v(t))\right\}\right) \mid \\
& =\left|(\Psi(t)-\Psi(a))^{1-\varrho}\left(\mathbf{I}_{a^{+}}^{\mu ; \Psi} f(t, u(t))-\mathbf{I}_{a^{+}}^{\mu ; \Psi} f(t, v(t))\right)\right| \\
& \leq \frac{(\Psi(t)-\Psi(a))^{1-\varrho}}{\Gamma(\mu)} \int_{a}^{t} \mathcal{L}_{\Psi}^{\mu}(t, \sigma)|f(\sigma, u(\sigma))-f(\sigma, v(\sigma))| d \sigma \\
& \leq \frac{L(\Psi(t)-\Psi(a))^{1-\varrho}}{\Gamma(\mu)} \int_{a}^{t} \mathcal{L}_{\Psi}^{\mu}(t, \sigma)|u(\sigma)-v(\sigma)| d \sigma \\
& \leq \frac{L \Gamma(\varrho)}{\Gamma(\mu+\varrho)}(\Psi(t)-\Psi(a))^{\mu}\|u-v\|_{\mathcal{P C}_{1-\varrho ; \Psi}(\mathcal{J}, \mathbb{R})} .
\end{aligned}
$$

From the choice of constant $L$, it follows that

$$
\|\mathcal{T} u-\mathcal{T} v\|_{\mathcal{P C}_{1-\varrho ; \Psi}(\mathcal{J}, \mathbb{R})} \leq \frac{1}{2}\|u-v\|_{\mathcal{P C}_{1-\varrho ; \Psi}(\mathcal{J}, \mathbb{R})} .
$$

Thus, $\mathcal{T}$ is a contraction and by the Banach contraction principle it has a unique fixed point in $\mathcal{B}_{r} \subseteq \mathcal{P C}_{1-\varrho ; \Psi}(\mathcal{J}, \mathbb{R})$ which is the unique solution of impulsive $\Psi$-HFDE (1.2)-(1.4).

\section{Nonlocal impulsive $\Psi$-HFDE}

In this section we examine the existence and uniqueness results for impulsive $\Psi$-HFDE with non local initial conditions given by

$$
\begin{aligned}
& { }^{H} \mathbf{D}_{a^{+}}^{\mu, \nu ; \Psi} u(t)=f(t, u(t)), t \in \mathcal{J} \backslash\left\{t_{1}, t_{2}, \ldots, t_{m}\right\}, \\
& \Delta \mathbf{I}_{a^{+}}^{1-\varrho ; \Psi} u\left(t_{k}\right)=\zeta_{k} \in \mathbb{R}, \quad k=1,2, \ldots, m, \\
& \mathbf{I}_{a^{+}}^{1-\varrho ; \Psi} u(a)+g(u)=\delta \in \mathbb{R},
\end{aligned}
$$

where $\mu, \nu, \varrho$ and the function $f$ are as given in the problem (1.2)-(1.4) and $g: \mathcal{P C} \mathcal{C}_{1-\varrho ; \Psi}(\mathcal{J}, \mathbb{R}) \rightarrow \mathbb{R}$ is a continuous function.

Theorem 6. (Existence) Assume that the function $f:(a, T] \times \mathbb{R} \rightarrow \mathbb{R}$ is continuous and satisfies the conditions $\left(A_{1}\right)-\left(A_{2}\right)$. Further, assume that $g$ : $\mathcal{P} \mathcal{C}_{1-\varrho ; \Psi}(\mathcal{J}, \mathbb{R}) \rightarrow \mathbb{R}$ is a continuous function that satisfies: 
$\left(A_{3}\right)|g(u)-g(v)| \leq L_{g}\|u-v\|_{\mathcal{P C}_{1-\varrho ; \Psi}(\mathcal{J}, \mathbb{R})}, \quad u, v \in \mathcal{P C}_{1-\varrho ; \Psi}(\mathcal{J}, \mathbb{R})$, with $0<$ $L_{g} \leq \frac{1}{6} \Gamma(\varrho)$.

Then, the nonlocal impulsive $\Psi-H F D E$ (5.1)-(5.3) has at least one solution in $\mathcal{P C} \mathcal{C}_{1-\varrho ; \Psi}(\mathcal{J}, \mathbb{R})$.

Proof. By applying the Lemma 3, the equivalent fractional integral equation of the nonlocal impulsive $\Psi$-HFDE (5.1)-(5.3) is given as follows

$$
u(t)=\Omega_{\Psi}^{\varrho}(t, a)\left(\delta-g(u)+\sum_{a<t_{k}<t} \zeta_{k}\right)+\mathbf{I}_{a^{+}}^{\mu ; \Psi} f(t, u(t)), t \in \mathcal{J} .
$$

Consider the set

$\mathcal{B}_{r^{*}}=\left\{u \in \mathcal{P C}_{1-\varrho ; \Psi}(\mathcal{J}, \mathbb{R}): \mathbf{I}_{a^{+}}^{1-\varrho ; \Psi} u(a)+g(u)=\delta,\|u\|_{\mathcal{P C}_{1-\varrho ; \Psi}(\mathcal{J}, \mathbb{R})} \leq r^{*}\right\}$, where

$$
r^{*} \geq 3\left(\frac{1}{\Gamma(\varrho)}\left\{|\delta|+G+\sum_{k=1}^{m}\left|\zeta_{k}\right|\right\}+\frac{\mathcal{M}}{\Gamma(\mu+1)}(\Psi(T)-\Psi(a))^{1-\varrho+\mu}\right),
$$

$G=|g(0)|$ and $\mathcal{M}=\sup _{\sigma \in \mathcal{J}}|f(\sigma, 0)|$. Define operators $\mathcal{R}$ and $\mathcal{Q}^{*}$ on $\mathcal{B}_{r^{*}}$ by

$$
\mathcal{R} u(t)=\Omega_{\Psi}^{\varrho}(t, a)\left(\delta-g(u)+\sum_{a<t_{k}<t} \zeta_{k}\right), \mathcal{Q}^{*} u(t)=\mathbf{I}_{a^{+}}^{\mu ; \Psi} f(t, u(t)), t \in \mathcal{J}
$$

Then the fractional integral equation (5.4) is equivalent to the operator equation

$$
u=\mathcal{R} u+\mathcal{Q}^{*} u, u \in \mathcal{P C}_{1-\varrho ; \Psi}(\mathcal{J}, \mathbb{R})
$$

We apply the Krasnoselskii's fixed point theorem (Theorem 3) to prove that the operator equation (5.5) has fixed point. Firstly, we show that $\mathcal{R} u+\mathcal{Q}^{*} v \in \mathcal{B}_{r^{*}}$ for any $u, v \in \mathcal{B}_{r^{*}}$. By assumptions $\left(A_{2}\right)$ and $\left(A_{3}\right)$, for any $u, v \in \mathcal{B}_{r^{*}}$ and $t \in \mathcal{J}$

$$
\begin{aligned}
& \left|(\Psi(t)-\Psi(a))^{1-\varrho}\left(\mathcal{R} u(t)+\mathcal{Q}^{*} v(t)\right)\right| \\
& =\left|(\Psi(t)-\Psi(a))^{1-\varrho}\left\{\Omega_{\Psi}^{\varrho}(t, a)\left(\delta-g(u)+\sum_{a<t_{k}<t} \zeta_{k}\right)+\mathbf{I}_{a^{+}}^{\mu ; \Psi} f(t, v(t))\right\}\right| \\
& \leq \frac{1}{\Gamma(\varrho)}\left(|\delta|+|g(u)|+\sum_{k=1}^{m}\left|\zeta_{k}\right|\right)+\frac{(\Psi(t)-\Psi(a))^{1-\varrho}}{\Gamma(\mu)} \int_{a}^{t} \mathcal{L}_{\Psi}^{\mu}(t, \sigma)|f(\sigma, v(\sigma))| d \sigma \\
& \leq \frac{1}{\Gamma(\varrho)}\left(|\delta|+|g(u)-g(0)|+|g(0)|+\sum_{k=1}^{m}\left|\zeta_{k}\right|\right) \\
& \quad+\frac{(\Psi(t)-\Psi(a))^{1-\varrho}}{\Gamma(\mu)} \int_{a}^{t} \mathcal{L}_{\Psi}^{\mu}(t, \sigma)|f(\sigma, v(\sigma))-f(\sigma, 0)| d \sigma \\
& \quad+\frac{(\Psi(t)-\Psi(a))^{1-\varrho}}{\Gamma(\mu)} \int_{a}^{t} \mathcal{L}_{\Psi}^{\mu}(t, \sigma)|f(\sigma, 0)| d \sigma
\end{aligned}
$$




$$
\begin{aligned}
& \leq \frac{1}{\Gamma(\varrho)}\left(|\delta|+L_{g}\|u\|_{\mathcal{P C}_{1-\varrho ; \Psi}(\mathcal{J}, \mathbb{R})}+G+\sum_{k=1}^{m}\left|\zeta_{k}\right|\right) \\
& \quad+\frac{L \Gamma(\varrho)}{\Gamma(\mu+\varrho)}(\Psi(t)-\Psi(a))^{\mu}\|v\|_{\mathcal{P} \mathcal{C}_{1-\varrho ; \Psi}(\mathcal{J}, \mathbb{R})}+\frac{\mathcal{M}(\Psi(t)-\Psi(a))^{1-\varrho+\mu}}{\Gamma(\mu+1)} \\
& \leq \frac{1}{\Gamma(\varrho)}\left(|\delta|+G+\sum_{k=1}^{m}\left|\zeta_{k}\right|\right)+\frac{L_{g}}{\Gamma(\varrho)} r^{*} \\
& \quad+\frac{L \Gamma(\varrho)}{\Gamma(\mu+\varrho)}(\Psi(T)-\Psi(a))^{\mu} r^{*}+\frac{\mathcal{M}(\Psi(T)-\Psi(a))^{1-\varrho+\mu}}{\Gamma(\mu+1)} .
\end{aligned}
$$

From the choice of $r^{*}, L$ and $L_{g}$, from the above inequality, we obtain

$$
\left\|\left(\mathcal{R} u+\mathcal{Q}^{*} v\right)\right\|_{\mathcal{P C}_{1-\varrho ; \Psi}(\mathcal{J}, \mathbb{R})} \leq r^{*} .
$$

Further, one can verify that $\mathbf{I}_{a^{+}}^{1-\varrho ; \Psi}\left(\mathcal{R} u+\mathcal{Q}^{*} v\right)(a)+g(u)=\delta$.

This shows that $\mathcal{R} u+\mathcal{Q}^{*} v \in \mathcal{B}_{r^{*}}$. Next, we prove that $\mathcal{R}$ is a contraction mapping. Let any $u, v \in \mathcal{B}_{r^{*}}$ and $t \in \mathcal{J}$. Consider

$$
\begin{aligned}
& \left|(\Psi(t)-\Psi(a))^{1-\varrho}(\mathcal{R} u(t)-\mathcal{R} v(t))\right| \\
& =\mid(\Psi(t)-\Psi(a))^{1-\varrho}\left\{\Omega_{\Psi}^{\varrho}(t, a)\left(\delta-g(u)+\sum_{a<t_{k}<t} \zeta_{k}\right)\right. \\
& \left.\quad-\Omega_{\Psi}^{\varrho}(t, a)\left(\delta-g(v)+\sum_{a<t_{k}<t} \zeta_{k}\right)\right\} \mid \\
& =\frac{1}{\Gamma(\varrho)}|g(u)-g(v)| \leq \frac{L_{g}}{\Gamma(\varrho)}\|u-v\|_{\mathcal{P C}} \| \varrho(\mathcal{J}, \mathbb{R}) .
\end{aligned}
$$

From the choice of $L_{g}$, we obtain

$$
\|R u-R v\|_{\mathcal{P C}_{1-\varrho ; \Psi}(\mathcal{J}, \mathbb{R})} \leq \frac{1}{6}\|u-v\|_{\mathcal{P C}_{1-\varrho ; \Psi}(\mathcal{J}, \mathbb{R})} .
$$

This shows that $\mathcal{R}$ is a contraction. The operator $\mathcal{Q}^{*}$ is compact and continuous as proved in the Theorem 4 . Hence by $\mathcal{P} \mathcal{C}_{1-\varrho ; \Psi}(\mathcal{J}, \mathbb{R})$-type Arzela-Ascoli Theorem $2 \mathcal{Q}^{*}$ is compact on $\mathcal{B}_{r^{*}}$. Further, as discussed in the proof of Theorem 4 the non local impulsive $\Psi$-HFDE (5.1)-(5.3) has at least one solution in $\mathcal{P C}_{1-\varrho ; \Psi}(\mathcal{J}, \mathbb{R})$.

Theorem 7. (Uniqueness) Assume that the function $f:(a, T] \times \mathbb{R} \rightarrow \mathbb{R}$ is continuous and satisfies the conditions $\left(A_{1}\right)-\left(A_{3}\right)$. Then, non local impulsive $\Psi-H F D E(5.1)-(5.3)$ has a unique solution in the space $\mathcal{P C}_{1-\varrho ; \Psi}(\mathcal{J}, \mathbb{R})$.

Proof. The proof can be completed following the same steps as in the proof the Theorem 5 .

\section{Applications}

By taking $\Psi(t)=t$ and $\nu \rightarrow 1$, the impulsive $\Psi$-HFDE (1.2)-(1.4) reduces to Caputo impulsive FDE of the form:

$$
{ }^{C} D_{a^{+}}^{\mu} u(t)=f(t, u(t)), t \in \mathcal{J} \backslash\left\{t_{1}, t_{2}, \ldots, t_{m}\right\},
$$




$$
\begin{aligned}
& \Delta u\left(t_{k}\right)=\zeta_{k} \in \mathbb{R}, k=1,2, \ldots, m, \\
& u(a)=\delta \in \mathbb{R},
\end{aligned}
$$

and we have the following existence and uniqueness theorems for Caputo impulsive FDE (6.1)-(6.3) as an applications of the Theorems 4 and 5.

Theorem 8. Assume that the function $f \in C(\mathcal{J}, \mathbb{R})$ satisfies the Lipschitz condition

$$
|f(t, u)-f(t, v)| \leq L|u-v|, t \in \mathcal{J}, u, v \in \mathbb{R}
$$

with $0<L \leq \frac{\Gamma(\mu+1)}{2(T-a)^{\mu}}$. Then, the Caputo impulsive FDE (6.1)-(6.3) has at least one solution in the space $\mathcal{P C}(\mathcal{J}, \mathbb{R})$.

Theorem 9. Under the suppositions of the Theorem 8 the impulsive Caputo FDE (1.2)-(1.4) has a unique solution in the space $\mathcal{P C}(\mathcal{J}, \mathbb{R})$.

\section{$7 \quad$ Examples}

In this section, we give examples to illustrate the utility of the results we obtained.

Example 1. Consider, the impulsive $\Psi$-HFDE

$$
\begin{aligned}
& { }^{H} \mathbf{D}_{0^{+}}^{\mu, \nu ; \Psi} u(t)=\frac{9}{5 \Gamma\left(\frac{2}{3}\right)}(\Psi(t)-\Psi(0))^{\frac{5}{3}}-\frac{(\Psi(t)-\Psi(0))^{4}}{16}+\frac{1}{16} u^{2}, t \in[0,1] \backslash\left\{\frac{1}{2}\right\}, \\
& \mathbf{I}_{0^{+}}^{1-\varrho ; \Psi} u(0)=0, \quad \Delta \mathbf{I}_{0^{+}}^{1-\varrho ; \Psi} u\left(\frac{1}{2}\right)=\sigma \in \mathbb{R},
\end{aligned}
$$

$0<\mu<1,0 \leq \nu \leq 1, \varrho=\mu+\nu-\mu \nu$ and $\Psi:[0,1] \rightarrow \mathbb{R}$ is as defined in preliminaries. Define the function $f:[0,1] \times[0,1] \rightarrow \mathbb{R}$ by

$$
f(t, u)=\frac{9}{5 \Gamma\left(\frac{2}{3}\right)}(\Psi(t)-\Psi(0))^{\frac{5}{3}}-\frac{(\Psi(t)-\Psi(0))^{4}}{16}+\frac{1}{16} u^{2} .
$$

Clearly, $|f(t, u)-f(t, v)| \leq \frac{1}{8}|u-v|, u, v \in \mathbb{R}, t \in[0,1]$. Thus $f$ satisfies the Lipschitz condition with the constant $L=\frac{1}{8}$. If the function $\Psi$ satisfies the condition

$$
L \leq \frac{\Gamma(\mu+\varrho)}{2 \Gamma(\varrho)(\Psi(1)-\Psi(0))^{\mu}},
$$

then problem (7.1)-(7.2) has unique solution. For instance, consider the particular case of the problem (7.1)-(7.2). By taking

$$
\Psi(t)=t, \mu=1 / 3 \text { and } \nu \rightarrow 1 .
$$

Then the problem (7.1)-(7.2) reduces to impulsive FDE involving Caputo fractional derivative operator of the form:

$$
{ }^{C} D_{0^{+}}^{\frac{1}{3}} u(t)=\frac{9}{5 \Gamma\left(\frac{2}{3}\right)} t^{\frac{5}{3}}-\frac{t^{4}}{16}+\frac{1}{16} u^{2}, t \in[0,1] \backslash\left\{\frac{1}{2}\right\},
$$




$$
\Delta u(1 / 2)=0, \quad u(0)=0 .
$$

Note that $\frac{\Gamma(\mu+\varrho)}{2 \Gamma(\varrho)(\Psi(T)-\Psi(a))^{\mu}}=\frac{1}{2} \Gamma\left(\frac{4}{3}\right) \approx 0.445$. Since $L=\frac{1}{8}$, the condition (7.3) is satisfied. Using the Theorem 4 with $\Psi(t)=t, a=0, T=1, \mu=\frac{1}{3}$ and $\nu \rightarrow$ 1 the problem (7.4)-(7.5) has a solution on $[0,1]$. By direct substitution one can verify that $u(t)=t^{2}$ is the solution of the problem (7.4)-(7.5).

Example 2. Consider an impulsive $\Psi$-HFDE

$$
\begin{aligned}
& H \mathbf{D}_{0^{+}}^{\mu, \nu ; \Psi} u(t)=\frac{\sin ^{4}(\Psi(t)-\Psi(0))}{((\Psi(t)-\Psi(0))+3)^{3}} \frac{|u(t)|}{1+|u(t)|}, t \in[0,1] \backslash\left\{\frac{1}{3}\right\}, \\
& \Delta \mathbf{I}_{0^{+}}^{1-\varrho ; \Psi} u(1 / 3)=\sigma, \quad \mathbf{I}_{0^{+}}^{1-\varrho ; \Psi} u(0)=\delta,
\end{aligned}
$$

where $0<\mu<1,0 \leq \nu \leq 1, \varrho=\mu+\nu-\mu \nu$. Define the function $f$ : $[0,1] \times[0,1] \rightarrow \mathbb{R}$ by $f(t, u)=\frac{\sin ^{4}(\Psi(t)-\Psi(0))}{((\Psi(t)-\Psi(0))+3)^{3}} \frac{|u|}{1+|u|}$. Let $u, v \in \mathbb{R}$ and $t \in[0,1]$. Then,

$$
\begin{aligned}
& |f(t, u)-f(t, v)|=\left|\frac{\sin ^{4}(\Psi(t)-\Psi(0))}{((\Psi(t)-\Psi(0))+3)^{3}} \frac{|u|}{1+|u|}-\frac{\sin ^{4}(\Psi(t)-\Psi(0))}{((\Psi(t)-\Psi(0))+3)^{3}} \frac{|v|}{1+|v|}\right| \\
& \leq \frac{1}{((\Psi(t)-\Psi(0))+3)^{3}}\left|\frac{|u|}{1+|u|}-\frac{|v|}{1+|v|}\right| \leq \frac{1}{((\Psi(1)-\Psi(0))+3)^{3}}|u-v| .
\end{aligned}
$$

This proves $f$ is Lipschitz function with the constant $L=\frac{1}{((\Psi(1)-\Psi(0))+3)^{3}}$. By Theorem 4 the problem (7.6)-(7.7) has a solution if

$$
\frac{1}{((\Psi(1)-\Psi(0))+3)^{3}} \leq \frac{\Gamma(\mu+\varrho)}{2 \Gamma(\varrho)(\Psi(1)-\Psi(0))^{\mu}} .
$$

\section{Future work}

In this paper, we have not investigated the continuous dependence on the various data and Ulam-Hyers stabilities of solution of (1.2)-(1.4), which is the point of our next investigation and will be published a future work.

Now, if we consider $\Psi(t)=t$ in the problem (1.2)-(1.4) with $A: D(A) \subset$ $X \rightarrow X$ generator of $C_{0}$-semigroup $\left(\mathbb{P}_{t \geq 0}\right)$ on a Banach space $X$, we have the following impulsive $\Psi$-HFDE with initial condition

$$
\begin{aligned}
{ }^{H} \mathbf{D}_{a^{+}}^{\mu, \nu} u(t) & =A u(t)+f(t, u(t)), t \in \mathcal{J} \backslash\left\{t_{1}, t_{2}, \ldots, t_{m}\right\} \\
\Delta \mathbf{I}_{a^{+}}^{1-\varrho} u\left(t_{k}\right) & =\zeta_{k} \in \mathbb{R}, k=1,2, \ldots, m, \\
\mathbf{I}_{a^{+}}^{1-\varrho ; \Psi} u(a) & =\delta \in \mathbb{R},
\end{aligned}
$$

with the same conditions of problem (1.2)-(1.4). The next step of the research is to analyze the problem (8.1)-(8.3). But the question may arise "Why not get the existence and uniqueness of mild solutions to the problem (8.1)-(8.3) with a $\Psi$-Hilfer fractional derivative operator ${ }^{H} \mathbf{D}_{a^{+}}^{\mu, \nu, \Psi}$ ? " The reason for noninvestigation with the $\Psi$-Hilfer fractional derivative comes from the fact of the 
non-existence of an integral transform, in particular, of Laplace with respect to another function, since it is an important condition in the investigation of the mild solution. Research in this sense has been developed and, in the near future, results can be published.

\section{Acknowledgements}

The first author acknowledges the Science and Engineering Research Board (SERB), New Delhi, India for the Research Grant (Ref: File no. EEQ/2018 /000407). The third author of this paper is financially supported by the PNPDCAPES scholarship of the Pos-Graduate Program in Applied Mathematics IMECC-Unicamp.

\section{References}

[1] S. Abbas, M. Benchohra, J.R. Graef and J. Henderson. Implicit fractional differential and integral equations: existence and stability. Walter de Gruyter GmbH \& Co KG, London, 2018. https://doi.org/10.1515/9783110553819.

[2] R. Agarwal, S. Hristova and D. ORegan. Some stability properties related to initial time difference for caputo fractional differential equations. Frac. Cal. Appl. Anal., 21(1):72-93, 2018. https://doi.org/10.1515/fca-2018-0005.

[3] R.P. Agarwal, M. Benchohra and B.A. Slimani. Existence results for differential equations with fractional order and impulses. Mem. Diff. Equ. Math. Phys, 44(1):1-21, 2008.

[4] B. Ahmad and S. Sivasundaram. Existence of solutions for impulsive integral boundary value problems of fractional order. Nonlinear Analysis: Hybrid Systems, 4(1):134-141, 2010. https://doi.org/10.1016/j.nahs.2009.09.002.

[5] A. Ali, K. Shah and D. Baleanu. Ulam stability results to a class of nonlinear implicit boundary value problems of impulsive fractional differential equations. Advances in Difference Equations, 2019(5):1-21, 2019. https://doi.org/10.1186/s13662-018-1940-0.

[6] Z. Bai, X. Dong and C. Yin. Existence results for impulsive nonlinear fractional differential equation with mixed boundary conditions. Boundary Value Problems, 2016(63), 2016. https://doi.org/10.1186/s13661-016-0573-z.

[7] D.D. Bainov and P.S. Simeonov. Impulsive differential equations: periodic solutions and applications. Longman Scientific and Technical Group Limited,New York, 1993.

[8] K. Balachandran and J.J. Trujillo. The nonlocal cauchy problem for nonlinear fractional integrodifferential equations in Banach spaces. Nonlinear Analysis: Theo. Meth. \& Appl., 72(12):4587-4593, 2010. https://doi.org/10.1016/j.na.2010.02.035.

[9] M. Benchohra and F. Berhoun. Impulsive fractional differential equations with variable times. Comput. Math. Appl., 59(3):1245-1252, 2010. https://doi.org/10.1016/j.camwa.2009.05.016.

[10] M. Benchohra, J. Henderson and S.K. Ntouyas. Impulsive differential equations and inclusions. Hindawi Publishing Corporation, Vol. 2, New York, 2006. 
[11] M. Benchohra and D. Seba. Impulsive fractional differential equations in Banach spaces. Elect. J. Qual. Theory Differ. Equ., 8(1), 2009. https://doi.org/10.14232/ejqtde.2009.4.8.

[12] M. Benchohra and B.A. Slimani. Existence and uniqueness of solutions to impulsive fractional differential equations. Elect. J. Diff. Equ., 2009(10):1-11, 2009.

[13] S. Das and P.K. Gupta. A mathematical model on fractional Lotka-Volterra equations. Theor. Biol., $\mathbf{2 7 7}(1): 1-6,2011$. https://doi.org/10.1016/j.jtbi.2011.01.034.

[14] E. Capelas de Oliveira and J.V.C. Sousa. Ulam-Hyers-Rassias stability for a class of fractional integro-differential equations. Results Math., 73(3):111, 2018.

[15] K. Diethelm. The analysis of fractional differential equation, Lecture notes in Mathematics. Springer, New York, 2010. https://doi.org/10.1007/978-3-64214574-2.

[16] S. Dipierro and E. Valdinoci. A simple mathematical model inspired by the Purkinje cells: from delayed travelling waves to fractional diffusion. Bull. Math. Biol., 80(7):1849-1870, 2018. https://doi.org/10.1007/s11538-018-0437-z.

[17] M. Feckan, Y. Zhou and J. Wang. On the concept and existence of solution for impulsive fractional differential equations. Commun Nonlinear Sci Numer Simulat., 17(7):3050-3060, 2012. https://doi.org/10.1016/j.cnsns.2011.11.017.

[18] T.L. Guo and W. Jiang. Impulsive fractional functional differential equations. Comput. Math. Appl., 64(10):3414-3424, 2012. https://doi.org/10.1016/j.camwa.2011.12.054.

[19] A.A. Kilbas, H.M. Srivastava and J.J. Trujillo. Theory and applications of fractional differential equations. Elsevier. Science, B.V., Amsterdam, 2006.

[20] K.D. Kucche, A.D. Mali and J.V.C. Sousa. On the nonlinear $\psi_{-}$ Hilfer fractional differential equations. Comp. Appl. Math., 38(73), 2019. https://doi.org/10.1007/s40314-019-0833-5.

[21] S. Kumar, A. Kumar and M.O. Zaid. A nonlinear fractional model to describe the population dynamics of two interacting species. Math. Meth. Appl. Sci., 40(11):4134-4148, 2017. https://doi.org/10.1002/mma.4293.

[22] Z. Liu and X. Li. Existence and uniqueness of solutions for the nonlinear impulsive fractional differential equations. Commun. Nonlinear Sci. Numer. Simulat., 18(6):1362-1373, 2013. https://doi.org/10.1016/j.cnsns.2012.10.010.

[23] G.M. Mophou. Existence and uniqueness of mild solutions to impulsive fractional differential equations. Nonlinear Analysis: Theory, Methods 83 Applications, 72(3-4):1604-1615, 2010. https://doi.org/10.1016/j.na.2009.08.046.

[24] S.G. Samko, A.A. Kilbas and O.I. Marichev. Fractional integrals and derivatives, theory and applications. Gordon and Breach, Yverdon, 1993.

[25] A.M. Samoilenko and N.A. Perestyuk. Impulsive differential equations. World Scientific, Singapore, 1995. https://doi.org/10.1142/2892.

[26] K. Shah, H. Khalil and R.A. Khan. Investigation of positive solution to a coupled system of impulsive boundary value problems for nonlinear fractional order differential equations. Chaos, Solitons \& Fractals, 77:240-246, 2015. https://doi.org/10.1016/j.chaos.2015.06.008.

[27] J.V.C. Sousa and E. Capelas de Oliveira. On the $\psi$-Hilfer fractional derivative. Commun. Nonlinear Sci. Numer. Simulat., 60:72-91, 2018. https://doi.org/10.1016/j.cnsns.2018.01.005. 
[28] J.V.C. Sousa and E. Capelas de Oliveira. On the Ulam-Hyers-Rassias stability for nonlinear fractional differential equations using the $\psi$-Hilfer operator. Fixed Point Theory and Appl., 20(3):96, 2018. https://doi.org/10.1007/s11784-0180587-5.

[29] J.V.C. Sousa and E. Capelas de Oliveira. Ulam-Hyers stability of a nonlinear fractional Volterra integro-differential equation. Appl. Math. Lett., 81:50-56, 2018. https://doi.org/10.1016/j.aml.2018.01.016.

[30] J.V.C. Sousa, E. Capelas de Oliveira and K.D. Kucche. On the fractional functional differential equation with abstract Volterra operator. Bull. Braz. Math. Soc., New Series, pp. 1-20, 2018.

[31] J.V.C. Sousa, E. Capelas de Oliveira and L.A. Magna. Fractional calculus and the ESR test. AIMS Math, 2(4):692-705, 2017. https://doi.org/10.3934/Math.2017.4.692.

[32] J.V.C. Sousa, K.D. Kucche and E. Capelas de Oliveira. Stability of $\psi$-Hilfer impulsive fractional differential equations. Appl. Math. Lett., 88:73-80, 2019. https://doi.org/10.1016/j.aml.2018.08.013.

[33] J.V.C. Sousa and E. Capelas Oliveira. A Gronwall inequality and the Cauchytype problem by means of $\psi$-Hilfer operator. Diff. Equ. $\&$ Appl., 11(1):87-106, 2019. https://doi.org/10.7153/dea-2019-11-02.

[34] J. Wang, K. Shah and A. Ali. Existence and Hyers-Ulam stability of fractional nonlinear impulsive switched coupled evolution equations. Math. Meth. Appl. Sci., 41(6):2392-2402, 2018. https://doi.org/10.1002/mma.4748.

[35] J. Wang, Y. Zhou and M. Fečkan. On recent developments in the theory of boundary value problems for impulsive fractional differential equations. Comput. Math. Appl., 64(10):3008-3020, 2012. https://doi.org/10.1016/j.camwa.2011.12.064.

[36] J. Wang, Y. Zhou and Z. Lin. On a new class of impulsive fractional differential equations. Appl. Math. Comput., 242:649-657, 2014. https://doi.org/10.1016/j.amc.2014.06.002.

[37] J.R. Wang, Y. Zhou and Fečkan. Nonlinear impulsive problems for fractional differential equations and Ulam stability. Comput. Math. Appl., 64(10):33893405, 2012. https://doi.org/10.1016/j.camwa.2012.02.021.

[38] W. Wei, X. Xiang and Y. Peng. Nonlinear impulsive integrodifferential equation of mixed type and optimal controls. Optimization, 55(1-2):141-156, 2006. https://doi.org/10.1080/02331930500530401.

[39] Y. Zhou. Basic theory of fractional differential equations. World scientific, 2014. https://doi.org/10.1142/9069. 